\title{
The Time has come turn on the Cutaneous Nerve System to manage Pain
}

\author{
Rob Sillevis*1 and Francisco Selva ${ }^{2}$ \\ ${ }^{1}$ Florida Gulf Coast University, USA \\ ${ }^{2}$ University of Valencia, Spain
}

*Corresponding author: Rob Sillevis, Florida Gulf Coast University, 10501 FGCU Boulevard, South Fort Myers, USA.

To Cite This Article: Rob Sillevis. The Time has come turn on the Cutaneous Nerve System to manage Pain. 2020 - 9(5). AJBSR.MS.ID.001430.

DOI: 10.34297/AJBSR.2020.09.001430.

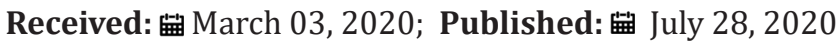

\section{Introduction}

Pain remains one the main reasons why people seek medical care. Most of the nociception leading to pain is typically generated in either the musculoskeletal system or the viscera. As the brain receives nociceptive information it will create an awareness of a noxious stimuli and respond accordingly [1]. The interconnection within the functioning of the brain, the immune system, and the health of the skin has been identified within the functioning of the brain, the immune system, and the health of the skin. Cutaneous sensory stimuli are transduced in the periphery by specialized organs or free nerve endings [2]. Primary afferent sensory neurons are long cells, often traversing distances of over $1 \mathrm{~m}$ in humans. Nociceptive and tactile stimulation can have profound neuroendocrine effects. Nociceptive information enters the central nervous system in an organized segmentally related fashion and can produce both peripheral and central sensitization. This is a state of hyperexcitability of nerve fibers, which react to stimuli weaker than the normal threshold, spreading to adjacent nerve fibers, producing prolonged repetitive electrical discharges from a single stimulus.

The skin is a highly complex organ, responsible for sensation, protection against the environment (contaminants, foreign proteins, infection) and, therefore, is linked to the immune and sensory systems. The skin, hypodermis, and superficial fascia are innervated by the cutaneous branches of an individual spinal nerve [3]. Epithelium cells are arranged is such a manner that they form a cohesive sheet. This function makes it perfect as the outside layer of the body's surface and as the lining of the digestive system. It originates from the ectoderm and endoderm. It consists of many layers and contains cells (such as lamellar cells, terminal Schwann cells, keratinocytes or Merkel cell) and nerves including free nerve endings (FNE). Although the most abundant cells of the epidermis are keratinocytes, there are also nonepithelial immune cells present in the epidermis, such as Langerhans cells and dendritic epidermal $\mathrm{T}$ cells [4]. Keratinocytes play an active role in the immune process. In response to skin damage or microbial intrusion, keratinocytes function as sentinels [5]. They are capable of initiating and amplifying a specific immune reaction through the activation of Langerhans cells that migrate to the lymph nodes [6]. The afferent sensory nerves are responsible for cutaneous perception of pressure, temperature, touch, and pain. Until recently, intra-epidermal FNE were considered exclusive cutaneous nociceptors, thermoreceptors, and proprioceptors [7]. This supported the idea that intra-epidermal nerve fibers were "free," and thus not associated with other cell types. The intra-epidermal FNE has traditionally been seen as the transducers of cutaneous noxious stimuli. However, there is a growing understanding how the keratinocyte actually contributes to cutaneous nociception. Talagas et al $[8,9]$ identified that the keratinocytes can initiate nociceptive responses.

Talagas et al. showed that FNE are located by keratinocytes. It appears that the keratinocyte cytoplasm is wrapped around the total length of the FNE, almost creating a tunnel for the FNE. It was concluded that the keratinocytes must play a role stimulating 
the FNE and therefore the transduction of nociceptive sensory information [8,9]. Findings demonstrate a clear relationship between the keratinocytes and nervous system. This was supported by the findings of Evdokimov et al [10]. They identified that patients with fibromyalgia have a higher interleukin 10 gene expression and this might result in cutaneous nociception. Gouin et al [11] identified that during cutaneous neurogenic inflammation the neuropeptides are released from sensory nerve endings and this will have an effect on the functioning of the keratinocyte.

It has been reported that there is a relationship between psychiatric conditions, such as depression and anxiety, and cutaneous hypersensitivity. The interaction between keratinocytes and nerve endings modulates pain and plays a role in the management of cutaneous hypersensitivity. The pathway by which this might occur is by the Keratinocytes maintaining homeostasis by activating endogenous glucocorticoids [12,13]. Cutaneous innervation innervation or immune components have been the focus of many medical treatments. Promising preliminary results have been obtained from a neuro-immunoendocrine approach with a focus on the cutaneous system. It has been identified that neuropeptides such as NGF, hormonal (vitamin D), anti-cytokine and capsaicin to attack the skin's sensory neurons [4,14-20].

The identification of keratinocytes as primary transducers of harmful stimuli is a paradigm shift in the field of cutaneous sensory transduction. By using the same afferent pathways, the FNE will send information to the spinal cord based on dermatomal relationships. In the cord non-noxious stimulation could aid in reducing a local central hyperexcitability. With other words stimulating the skin causing physical ionization through the receptors seems to be a direct, fast and effective way to down regulate the nervous system as a whole will result in decreased pain and improved ROM. It is time we shift focus and turn on the cutaneous nerve system to manage pain.

\section{Reference}

1. O'Sullivan RL, Lipper G, Lerner EA (1998) The neuro-immunocutaneous-endocrine network: relationship of mind and skin. Archives of dermatology 134(11): 1431-1435.

2. Clark AJ, Menendez G, AlQatari M (2018) Functional imaging in microfluidic chambers reveals sensory neuron sensitivity is differentially regulated between neuronal regions. Pain 159(7): 1413-1425.

3. Rice FL, Castel D, Ruggiero E (2019) Human-like cutaneous neuropathologies associated with a porcine model of peripheral neuritis: A translational platform for neuropathic pain. Neurobiol Pain 5: 100021.

4. Jankowski MP, Baumbauer KM, Wang T, Albers KM, Davis BM, et al. (2017) Cutaneous neurturin overexpression alters mechanical, thermal, and cold responsiveness in physiologically identified primary afferents. J Neurophysiol 117(3): 1258-1265.

5. Kimber I, Cumberbatch M (1992) Stimulation of Langerhans cell migration by tumor necrosis factor alpha (TNF-alpha). J Invest Dermatol 99(5): 48S-50S

6. Black AP, Ardern Jones MR, Kasprowicz V (2007) Human keratinocyte induction of rapid effector function in antigen-specific memory CD4+ and CD8+ T cells. Eur J Immunol 37(6): 1485-1493.

7. Abraira VE, Ginty DD (2013) The sensory neurons of touch. Neuron $79(4): 618-639$.

8. Talagas M, Lebonvallet N, Berthod F, Misery L (2019) Cutaneous nociception: Role of keratinocytes. Exp Dermatol 28(12): 1466-1469.

9. Talagas M, Lebonvallet N, Misery L (2018) Intraepidermal nerve fibres are not the exclusive tranducers of nociception. J Neurosci Methods 306: 92-93.

10. Evdokimov D, Kress L, Dinkel P, Frank J, Sommer C, et al. (2020) Painassociated Mediators and Axon Pathfinders in Fibromyalgia Skin Cells. J Rheumatol 47(1): 140-148.

11. Gouin O, L'Herondelle K, Lebonvallet N (2017) TRPV1 and TRPA1 in cutaneous neurogenic and chronic inflammation: pro-inflammatory response induced by their activation and their sensitization. Protein Cell 8(9): 644-661.

12. Jafferany M, Davari ME (2019) Itch and psyche: psychiatric aspects of pruritus. International journal of dermatology 58(1): 3-23.

13. Matsumoto A, Murota H, Terao M, Katayama I (2018) Attenuated Activation of Homeostatic Glucocorticoid in Keratinocytes Induces Alloknesis via Aberrant Artemin Production. J Invest Dermato 138(7): 1491-1500.

14. Drummond PD (2014) Neuronal changes resulting in up-regulation of alpha-1 adrenoceptors after peripheral nerve injury. Neural Regen Res 9(14): 1337-1340.

15. Drummond PD, Dawson LF, Finch PM, Drummond ES, Wood FM, et al. (2015) Up-regulation of cutaneous alpha1-adrenoceptors after a burn. Burns 41(6): 1227-1234.

16. Yamaguchi J, Aihara M, Kobayashi Y, Kambara T, Ikezawa Z, et al. (2009) Quantitative analysis of nerve growth factor (NGF) in the atopic dermatitis and psoriasis horny layer and effect of treatment on NGF in atopic dermatitis. J Dermatol Sci 53(1): 48-54.

17. Barrea L, Savanelli MC, Di Somma C (2017) Vitamin D and its role in psoriasis: An overview of the dermatologist and nutritionist. Reviews in endocrine \& metabolic disorders 18(2): 195-205.

18. Bernstein JE, Parish LC, Rapaport M, Rosenbaum MM, Roenigk HH, et al. (1986) Effects of topically applied capsaicin on moderate and severe psoriasis vulgaris. J Am Acad Dermatol 15(3): 504-507.

19. Ellis CN, Berberian B, Sulica VI (1993) A double-blind evaluation of topical capsaicin in pruritic psoriasis. J Am Acad Dermatol 29(3): 438442.

20. Gupta R, Gupta M, Mangal S, Agrawal U, Vyas SP, et al. (2016) Capsaicinloaded vesicular systems designed for enhancing localized delivery for psoriasis therapy. Artif Cells Nanomed Biotechnol 44(3): 825-834. 